\title{
New Immunotherapy Biomarker N-Cadherin Rescue Immunosuppress Caused by PD-L1/IDO-1 in Prostate Cancer
}

\section{Yi Sun}

Duke University

Ju Guo

First Affiliated Hospital of Nanchang University

Robert E Reiter

UCLA

\section{Huan Xu}

Shanghai Jiao Tong University

Jiao Huang ( $\nabla$ huang.duke@hotmail.com )

Duke University School of Medicine https://orcid.org/0000-0003-0192-5825

\section{Qiang Wei}

Sichuan University West China Hospital

\section{Lu Yang}

Sichuan University West China Hospital

\section{Research}

Keywords: N-cadherin, PD-L1, IDO-1, EMT, Immunosuppression, Prostate cancer

Posted Date: July 17 th, 2020

DOl: https://doi.org/10.21203/rs.3.rs-42523/v1

License: (c) (i) This work is licensed under a Creative Commons Attribution 4.0 International License. Read Full License 


\section{Abstract}

Background: N-cadherin is a major regulatory factor of epithelial-to-mesenchymal transition (EMT), and the association and coordination of EMT and neuroendocrine differentiation (NED) with immunosuppression have been reported in several tumor types. Therefore, we hypothesize that $\mathrm{N}$ cadherin may play vital role in this process.

Methods: We performed human tissue studies, cell-level explorations and in vivo experiments to determine the statuses of $\mathrm{N}$-cadherin and immune checkpoint proteins (PD-L1 and IDO-1) to verify the coexpression of these biomarkers. Then, we used tumor-bearing mice to test the treatment effect of $\mathrm{N}$ cadherin deletion. Moreover, the mechanism by which N-cadherin regulates PD-L1/ID 0-1 expression was also assessed.

Results: According to our data, N-cadherin expression is necessary for PD-L1 expression and dramatically increases the expression of ID 0-1 through an IFN- $囚$ response. On the contrary, IDO-1 and PD-L1 can prompt EMT. N-cadherin knockout completely reverses this process. The core mechanism underlying these phenomena is that $\mathrm{N}$-cadherin causes JAK1 to be re-expressed in cells with genomic JAK1 loss via the active EMT/immunosuppression positive feedback loop.

Conclusion: These data indicate that $\mathrm{N}$-cadherin can regulate the EMT/immunosuppression positive feed loop. Preclinical research has shown that $\mathrm{N}$-cadherin deletion promotes the development of antitumor responses by decreasing immunosuppression.

\section{Background}

Hormone treatment can induce the conversion of androgen-dependent prostate tumors into androgenindependent tumors, and a high proportion of castration-resistant prostate cancer (CRPC) cases eventually progresses to highly aggressive small cell neuroendocrine prostate cancer (SCNC) with a poor prognosis $\left[{ }^{1,2,3}\right]$. Moreover, immunotherapy has emerged as a valuable strategy for several tumor types [ ${ }^{4}$, 5], and thus, researchers have attempted to apply immunotherapy in prostate cancer especially in CRPC and SCNC; however, it is first necessary to find a treatment-related biomarker. The immune checkpoint protein programmed death ligand-1 (PD-L1) has been shown to induce T lymphocyte anergy and/or apoptosis by binding to its receptor PD-1, and some research has indicated that PD-L1 expression may be a reason tumor cells can escape immune surveillance, which leads to tumor development and metastasis $\left.{ }^{6}\right]$. In addition to PD-L1, indole amine 2,3-dioxygenase (IDO-1) is another checkpoint protein that contributes to cancer immune evasion. IDO-1 is a tryptophan-catabolizing enzyme that can induce the rapid conversion of tryptophan (Try) into kynurenine (Kyn) $\left[{ }^{7}\right]$, and can activate regulatory $T$ (Treg) cells to suppress effector T cells [ $\left.{ }^{8}\right]$. Both PD-L1 and IDO-1 can contribute to immune system impairment in individuals with cancer. Thus, inhibition of the immunosuppressive factors ID 0-1 and PD-L1 has been proposed as an immunotherapy strategy. 
In addition, the mutual positive feedback between epithelial-mesenchymal transition (EMT) and immunosuppression has also been demonstrated in several cancers $[9,10,11,12,13,14,15]$. EMT refers to the loss of polarity, tight junctions and adhesion between epithelial cells and the acquisition of infiltration and migration abilities $\left[{ }^{16}\right]$. Tumor cells, which undergo EMT, can invade normal tissues and induce metastasis, and a recent study demonstrated that $\mathrm{N}$-cadherin can regulate EMT status $\left[{ }^{12}\right]$. Therefore, we proposed the hypothesis that $\mathrm{N}$-cadherin can improve EMT and then enhance the positive feedback loop of EMT/immunosuppression, and that N-cadherin can be considered a therapeutic target. In this study, we attempted to address this issue and propose a potential new treatment strategy for advanced prostate cancer.

\section{Methods}

\section{Patients and tissue samples}

Several tissue microarrays (TMAs) were constructed and have been previously reported $\left[{ }^{17}\right]$. TMAs containing both benign and cancer tissues were constructed with prostatectomy specimens from 30 patients with localized prostate adenocarcinoma. Three cores were obtained from the benign and tumor areas of each prostatectomy specimen and were incorporated into the TMA. A CRPC TMA was constructed using CRPC tissue samples from 18 patients who were histologically diagnosed with adenocarcinoma but failed to respond to hormonal therapy. These tissue samples were derived from tissue collected during transurethral resection for the relief of obstructive symptoms and were incorporated into the CRPC TMA. A small cell prostate cancer (SCC) TMA was constructed from 16 primary SCC cases collected at Duke University Hospital. All samples were collected from patients who provided informed consent, and all related procedures were performed with the approval of the internal review and ethics boards of the indicated hospitals.

\section{Immunohistochemistry in tissue samples}

To prepare them for immunohistochemistry (IHC), all the slides (including the target sample slides and the positive and negative control slides) were deparaffinized, rehydrated, and boiled in a water bath for 40 min in citrate buffer ( $\mathrm{pH}$ 6.0) before antibody staining. Then, the slides were incubated with primary antibodies (the optimized dilution was previously determined) for $1 \mathrm{~h}$ at room temperature. Horseradish peroxidase-conjugated secondary antibodies (Dako EnVision+Kit) were applied for $30 \mathrm{~min}$ and visualized with diaminobenzidine (DAB) after incubation for $30 \mathrm{~min}$ at room temperature.

\section{Cell culture and cell treatment}

The benign prostate cell line BPH-1 and the prostate cancer cell lines LNCap, C4-2, CWRR-1, PC3 and NCIH660 were obtained from ATCC. The LASCPC-01 cell line was obtained from Owen N. Witte's laboratory $\left[{ }^{18}\right]$, The NC-IH660 and LASCPC-01 cells were cultured in HITES medium, while the LNCap, C4-2, CWRR-1 and PC3 cells were cultured in RPMI medium supplemented with $10 \%$ fetal bovine serum (FBS) and $1 \%$ 
penicillin, and BPH-1 cells were cultured in RPMI medium supplemented with $20 \% \mathrm{FBS}$ and $1 \%$ penicillin. The LNCap C1/C2/C3/LC1 cell lines and the N-cad knockdown PC3 cell line (PC3/N-cad KD) were obtained from Robert E Reiter's laboratory. These cell lines were also cultured in RPMI medium supplemented with $10 \% \mathrm{FBS}$ and $1 \%$ penicillin. All cell lines were maintained at $37 \mathrm{C}$ in $5 \% \mathrm{CO}$. When we tested the effects of interferon gamma (IFN- - ), a JAK/STAT inhibitor and Kyn on the cells, we treated cells

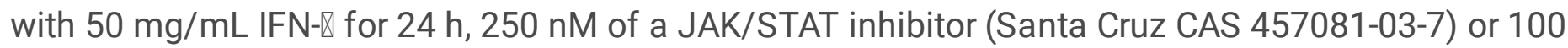
Imol/L Kyn in the medium for $48 \mathrm{~h}$. We also treated the cells with a PD-L1 neutralizing antibody (10 $\mu \mathrm{g} / \mathrm{mL}$ ) (Invitrogen, 16-5982-82) for $24 \mathrm{~h}$ to inhibit PD-L1 expression [ $\left.{ }^{19}\right]$. IL-8 inhibition was previously described [ $\left.{ }^{20}\right]$, briefly, cells were transfected with $100 \mathrm{nM}$ DNA plasmids with a short hairpin RNA to inhibit IL-8, (category number C01001; GenePharma, Shanghai, China) using the transfection reagent Lipofectamine 3000 (Invitrogen, Carlsbad, CA) according to the manufacturer's instructions. Protein was extracted with RIPA buffer, and RNA was extracted with TRIzol.

\section{Antibodies}

The antibodies used for both IHC and Western blotting were anti-IDO-1 (CST-51851), anti-interferon gamma receptor 1 (IFNGR1; Invitrogen A5-27841), anti-p-IFNGR1 (Invitrogen PA5-38504), and anti-N-cad (Thermo Fisher MA1-91128 CH-19). The PD-L1 antibody used for Western blotting and flow cytometry was CST-13684, and the one used for IHC was Dako Phe19-Thr239. The secondary antibody used for flow cytometry was conjugated with Alexa Fluor 488. Western blotting for JAK1, p-JAK1, STAT1, p-STAT1, STAT3 p-STAT3, E-cadherin, and Vimentin was performed using the following antibodies: Santa Cruz SC1677, PA5-104554, Santa Cruz SC-464, Invitrogen KIKSI0803, Santa Cruz SC-8019, CST-9145, Sigma SAB2104222 and Sigma V6389, respectively. IHC for IFN- \ was performed with the A259336 antibody purchased from Invitrogen. The PCR primers are shown in the Supplemental Materials.

\section{Tumor beard mice experiments}

Immunocompromised NSG (NOD.Cg-Prkdc $\| 2 \mathrm{rg} / \mathrm{SzJ}$ ) and nude (nu/nu) mice were obtained from The Jackson Laboratory. In all, $2 \times 106$ LNCap (and LNCap C1) or 1×106 PC3 (and PC3 N-cad KO) cells were suspended in $0.1 \mathrm{~mL} 1 \times$ RPMI 1640 medium and 10\% FBS with 50\% Matrigel (Corning) and then inoculated subcutaneously into the bilateral flanks of 3- to 6-week-old male NSG mice. After the LNCap and PC3 xenografts grew to $1 \mathrm{~cm}^{3}$ (tumor volume was calculated using the formula volume $(\mathrm{mm} 3)=$ length $\times$ height2/2), the mice were divided into two groups. Each mouse in one group was treated with 10

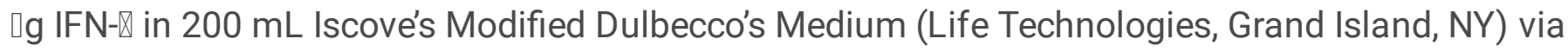
intraperitoneal injection once per day for two consecutive days. Each mouse in the other group received DMSO as a control. Forty-eight hours after IFN- $\$ or DMSO injection, the mice were sacrificed and the xenografts were harvested, after which they were fixed in $10 \%$ formalin and embedded in paraffin for subsequent analysis. Single-cell insolation was performed with the xenografts; the tissue samples were completely minced and incubated in an enzymatic mixture (80 mg collagenase type II in $40 \mathrm{~mL}$ advanced DMEM with 1/1000 ROCK inhibitor and 1/1000 1.0 M DHT) for overnight digestion. The cells were washed with fresh advanced DMEM before culture in $5 \mathrm{~mL}$ TrypLE+5 $\mathrm{LL}$ ROCK inhibitor in a $37^{\circ} \mathrm{C}$ 
incubator for $5 \mathrm{~min}$. The cells were repeatedly pipetted up and down and cultured again to efficiently separate the clumps. The cells were then centrifuged and stored in PBS for flow cytometric analysis or RNA extraction.

\section{Quantification and statistical analysis}

Statistical analyses were performed using R Project for Statistical Computing, Statistica, GraphPad, and MATLAB. The details and analytical methods are indicated in the figure legends, Results, or Methods sections. The immunohistochemically stained slides were scanned using an Aperio AT2 microscope (Leica Biosystems) at 20x magnification and were analyzed with Aperio software. Quantification was performed by pathologists who were blinded to the diagnosis of the tissue cores. Flow cytometric analysis was performed using BD FACSDiva software, and the results were analyzed using FlowJo 10.0. A human cytokine array was quantified using ImageJ.

\section{Results}

\section{Expression of the immunosuppressive factors PD-L1/IDO-1 is associated with N-cadherin expression}

Some studies have demonstrated that neuroendocrine differentiation (NED) and EMT share similar properties and are always co-expressed in advanced cancer $[21,22,23,24,25]$. To determine whether the EMT marker $\mathrm{N}$-cadherin is also specifically expressed in NE cells in human prostate cancer and to address the role of $\mathrm{N}$-cadherin in the expression of immunosuppressive factors, we performed immunohistochemistry $(\mathrm{IHC})$ on TMAs containing human prostate cancer samples of different stages using antibodies against $\mathrm{N}$-cadherin. Scattered or clustered NE cells expressing $\mathrm{N}$-cadherin were identified (Fig. 1a), and according to the IHC score (Fig. 1b), the expression of N-cad was found only in NE tissue samples, but not in benign, adenocarcinoma or CRPC tissue samples. We also performed a cell-level experiment for which we selected different cell lines to represent the different types of prostate cancer. $\mathrm{BPH}-1$ is a benign prostate cell line, and LNCap is an early-stage tumor cell line that is usually representative of adenocarcinoma. C4-2 and CWRR1 cells are commonly used to represent the CRPC phenotype. PC3, LASCPC-01 and $\mathrm{NCIOH660}$ cells were selected as NE cell lines. As shown in the results in Fig. 1c, the cell lines with $\mathrm{N}$-cadherin expression (PC3, LASCPC and $\mathrm{NCl}-\mathrm{H} 600$ ) also expressed low levels of E-cadherin, which is in agreement with published data; this demonstrates that $\mathrm{N}$-cadherin can enhance EMT [ ${ }^{12}$ ]. Interestingly, as shown in Fig. 1d, we confirmed that PD-L1 was observed only in tissue with Ncadherin positivity (SCC group). The analysis of the PD-L1 expression signature is shown in Fig. 1e, which indicates that the IHC score for PD-L1 in the SCC group was much higher than the scores in the other groups. Fig. $1 f$ and S-Fig. $1 a$ and $b$ also show the protein and mRNA data for both PD-L1 and IDO-1; only in the cell lines in which N-cadherin is activated was PD-L1 expression detectable, and IDO-1 expression was also increased in those cells. In addition, when the expression of ID0-1 was compared among the different tissue groups according to the IHC results, the highest level of IDO-1 was found in the $\mathrm{N}$-cadherin-positive group (Fig. $1 \mathrm{~g}$ and $\mathrm{h}$ ). Fig. 1i shows the synergistic association between PD-L1 and ID 0-1. Fig. $1 \mathrm{j}$ and $\mathrm{k}$ provide more evidence that the expression of PD-L1 and ID O-1 was higher in the N- 


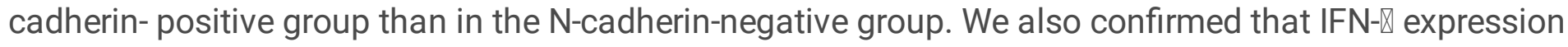

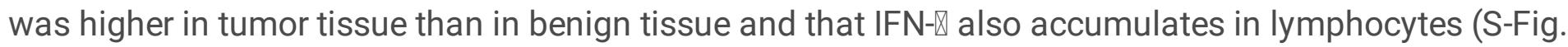
1c), where it simulates the tumor microenvironment. This explains why we selected IFN- $\$ to determine the mechanism of N-cadherin mediation of PD-L1/IDO-1 production.

To better characterize the impact of $\mathrm{N}$-cadherin on immunosuppression, we also performed an in vivo experiment to test the association between $\mathrm{N}$-cadherin and immunosuppression. We first implanted tumor cells in an inguinal site (Fig. 2a): PC3 cells were used in one group, while LNCap cells were used in the other group. Each group received IFN- $\triangle$ as a treatment or DMSO as a control after the tumors grew to $1 \mathrm{~cm}^{3}$; we collected the tumors $48 \mathrm{~h}$ after drug injection (the animals were sacrificed by $\mathrm{CO}_{2}$ inhalation). Some tissue was used for paraffin embedding, some was dissociated into single cells for flow cytometric analysis, while other tissue was used for RNA extraction for PCR. The expression of PD-L1 was examined by flow cytometry and was inducible only in the PC3 group (Fig. 2b). Evaluation by IHC revealed that only the PC3 tumor-bearing mice could produce PD-L1/IDO-1 under IFN- $\$ stimulation (Fig. 2c and d). The same results were obtained with PCR (Fig. 2e and f). Interestingly, as shown in Fig. 2g, N-cadherin expression was found only in the PC3 group and was enriched after response to IFN- $\mathrm{X}$. Additionally, Fig. $2 \mathrm{~h}$ shows hematoxylin and eosin (H\&E) staining of mouse tissue.

Overall, we reported that the enhanced accumulation of $\mathrm{N}$-cadherin is linked with higher expression of the checkpoints molecules PD-L1/IDO-1. Confirmation that EMT and immunosuppression are able to mutually modulate each other was our next area of focus.

\section{How EMT and immunosuppression mutually regulate each other}

The increased production of $\mathrm{N}$-cadherin under IFN- $₫$ stimulation was identified by IHC (Fig. $2 \mathrm{~g}$ ). To dissect the mechanism, we used IFN- $₫$ to treat cell lines that represent benign prostate tissue and different prostate cancer types, and then evaluated the expression of the EMT markers N-cadherin, E-cadherin and

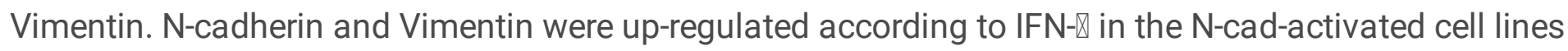
(Fig. 3a), while E-cadherin expression was down-regulated. However, IFN- $\triangle$ did not cause N-cad-

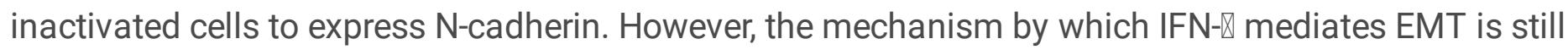
unclear and whether N-cadherin is able to regulate the PD-L1 and IDO-1 expression signature is unknown. Therefore, we tested whether ID 0-1 can change the EMT status in these cells. IDO-1 is an enzyme involved in Try metabolism that improves Try conversion into Kyn. Hence, using Western blot, we detected whether local accumulation of Kyn enhances the EMT process. Stimulation with $100 \mathrm{mmol} / \mathrm{L} \mathrm{Kyn}$ significantly increased the expression of $\mathrm{N}$-cadherin and Vimentin, while the expression of E-cadherin decreased (Fig. 3b). This result is in agreement with the findings reported by Kolijn et al $\left.{ }^{26}\right]$. Additionally, we found that Kyn and IFN- $₫$ treatment failed to accelerate cell growth (S-Fig-2a), and thus the phenotypic change resulted directly from stimulation instead of rapid cell growth. These results may also explain why IDO-1 and PD-L1 expression was simultaneously enriched (Fig. 1i). Another interesting finding is that PD-L1 expression also improved after Kyn treatment, while ID0-1 production did not significantly change (Fig. 3b), which contrasts with the results reported by Kolijn et al. ${ }^{[26}$. Indeed, after treatment with a PD-L1 
neutralizing antibody, we tested the EMT status according to N-cadherin and Snail expression. The EMT activation level was reduced after PD-L1 attenuation (Fig. 3c). These data indicated that the IFN- $\square$ response can not only induce immunosuppression but can also promote EMT.

Then, we compared the phenotypes between $\mathrm{N}$-cadherin-overexpressing and $\mathrm{N}$-cadherin knockout cells. As shown in Fig. 3d, LNCap C1 and C2 cells concomitantly lost E-cadherin expression and gained Vimentin expression, although one cell line with low expression (LNCap subline (C3)) retained E-cadherin expression and did not change morphologically (although we observed N-cadherin expression in LNCap C3 cells, E-cadherin was also expressed and appeared to exhibit a stronger expression than $\mathrm{N}$-cad); thus,

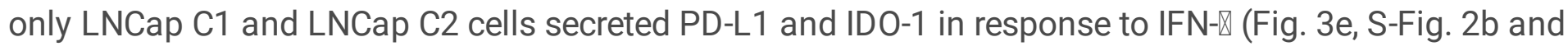
c). The LNCap N-cad-activated cell lines LNCap C1 and C2 were characterized by a significant upregulation in growth compared with the $\mathrm{C} 3$ cell line (S-Fig. 3a). This phenomenon also reminds us of the accelerated growth in $\mathrm{N}$-cadherin activated cells. At the same time, $\mathrm{N}$-cadherin knockout reversed NED and EMT to some degree, and IL-8 expression disappeared, which can explain this phenomenon seen in our data (S-Fig. 3b) and agrees with findings of previous studies [ $\left.{ }^{12,13,15}\right]$. After $\mathrm{N}$-cadherin was attenuated, PD-L1 and IDO-1 expression almost disappeared (Fig. 3e and S-Fig. 2b and C). Additionally, we did not observe Vimentin expression in LNCap C3 cells, and thus, we still did not consider this cell line to be an EMT-activated cell line. This may be the reason that PD-L1 and IDO-1 failed to be induced in LNCap C3 cells (Fig. 3e and S-Fig. 2b and c).

Since we proposed that N-cadherin can be considered a therapeutic target, we wanted to acquire more in vivo evidence, and thus, we constructed a new tumor-bearing mouse model. We implanted PC3 N-cad KO cells and LNCap N-cad-activated cells into NOD scid gamma (NSG) mice. The tumor growth status was

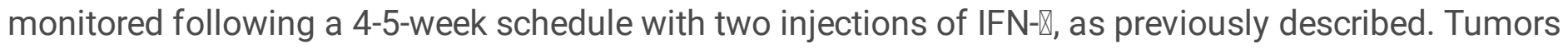

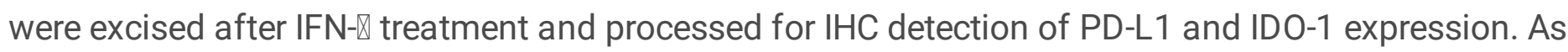
shown in Fig. 3f, Fig. 3g and Fig. 3h, for the LNCap C1 tumor sample, under stimulation with IFN-区, the expression of PD-L1 and IDO-1 was detectable, while after N-cadherin knockout, PC3-derived tumors were no longer able to express PD-L1 and IDO-1. The anti-immunosuppressive effect of N-cadherin was identified by comparison of those models. Indeed, the results of S-Fig. 3c suggest rapid tumor growth in the N-cadherin-activated LNCap mouse model, whereas in contrast, the N-cad KO PC3 model was characterized by significant down-regulation of tumor growth.

\section{The IFNGR/JAK/STAT pathway is responsible for the expression of PD-L1 and IDO-1}

We examined the mechanism of PD-L1 and IDO-1 expression as well as the role of N-cadherin in this process. First, we focused on IFNGR1 because IFN- $₫$ primarily regulates PD-L1 and IDO-1 expression through IFNGR1 [ $\left.{ }^{27}\right]$. The IHC results verified our hypothesis, as IFNGR1 expression was significantly higher in the SCC group, even though some staining was also seen in the CRPC group (Fig. $4 a$ and b). We also compared IFNGR1 expression in N-cadherin-positive cases and N-cadherin-negative cases, and the results showed IFNGR1 enrichment in N-cadherin-positive tissues (Fig. 4c). Similar results were observed for p-IFNGR1, which is the activated form of IFNGR1, with the highest level in the SCC group (Fig. 4d and 
e). p-IFNGR1 was also up-regulated in the N-cadherin-positive cases (Fig. 4f). Additionally, IHC in tissue from tumor-bearing mice showed that IFNGR1 staining was found only in the PC3 group, and only in this group was IFNGR1 able to be activated by IFN- (Fig. 4g and h). Fig. 4i and Supplemental Fig-4a demonstrated that at the cellular level, the expression of IFNGR1 is higher in N-cadherin-activated cell lines and is able to be activated by IFN- $囚$ only in the CRPC and SCC cell lines ( $\mathrm{N}$-cadherin-activated cell

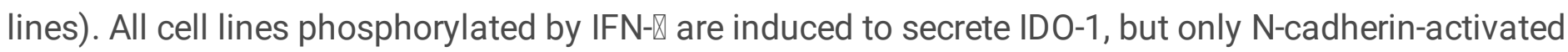
cell lines, which contain higher levels of p-IFNGR1, are able to produce PD-L1 (Fig. 1f).

We blocked this pathway to better understand the IFNGR-related regulatory process. Generally, IFNGR activates the JAK/STAT pathway, which leads to PD-L1 and IDO-1 production. Therefore, we blocked the entire JAK/STAT pathway to evaluate whether IDO-1 and PD-L1 could still be induced. As shown in Fig. 4a, PD-L1 and IDO-1 were no longer expressed when the JAK/STAT pathway was blocked. We confirmed that PD-L1 and IDO-1 are mediated by the JAK/STAT pathway, and therefore, we then explored the impact of different JAK/STAT pathway molecules on the expression of PD-L1 and IDO-1. Generally, PD-L1 and IDO-1 can be regulated by STAT1 or STAT-3, and thus we tested the total and phosphorylated STAT1/3 levels. We found that JAK1 was expressed and was activated only in NE cell lines and that STAT1 was activated only in those cell lines (Fig. 5b and S-Fig. 4a), even though STAT1 is expressed in all cells (Fig. $5 \mathrm{c}$ and S-Fig. 4a). In addition, when IDO-1 was expressed in CRPC cell lines, it was not regulated by STAT1, but rather, by STAT3, as expected, which was confirmed by the STAT3 and p-STAT3 data; in CRPC cells, STAT3 was phosphorylated in response to IFN- (Fig. 5d). This also indicated that STAT3 cannot determine PD-L1 expression. Another interesting phenomenon is that although STAT3 is not expressed in PC3 cells, PC3 cells were still able to secrete IDO-1 and PD-L1 (Fig. 5d), which indicated that the JAK1/STAT1 pathway can regulate the expression of both PD-L1 and IDO-1. In addition, STAT3 is constitutively activated in the $\mathrm{NCl}-\mathrm{H} 660$ cell line, which is why IDO-1 is expressed in these cells without any treatment, while PD-L1 is not. We also tested the impact of Kyn on the JAK/STAT pathway and found that Kyn can up-regulate the phosphorylation of JAK1, STAT1 and STAT3 even though the total protein levels did not change. These results indicated that IDO-1 can increase JAK/STAT pathway activation and the expression of PD-L1 (Fig. 5e). Based on this result and the data shown in Fig. 1i, this can be considered an explanation for the findings of other studies that demonstrated that PD-L1 and ID 0-1 are always co-expressed in settings of immunosuppression $\left[{ }^{27}\right]$. Then, we compared JAK1 expression between $\mathrm{N}$-cadherin-overexpressing and $\mathrm{N}$-cadherin $\mathrm{KO}$ cell lines to explore why PD-L1/IDO-1 can be induced in LNCap C1 and LNCap C2 cells. Fig. 5f and S-Fig. 4b show that JAK1 was expressed in LNCap C1 and LNCap C2 cells but not in LNCap C3 cells, and that N-cadherin KO in PC3 cells caused a decrease in JAK1 and p-JAK1 expression. Examination of the phosphorylated protein levels (Fig. $5 \mathrm{~g}$ ) demonstrated that the JAK/STAT pathway in LNCap C1 and C2 cells can be activated by IFN-囚, which supports the data in Fig. $5 f$.

\section{Discussion}


We systematically profiled cells in vitro, cells in in vivo models, and preclinical therapy to reveal a potential new targeted treatment strategy involving $\mathrm{N}$-cadherin and the molecular mechanism by which $\mathrm{N}$ cadherin regulates immunosuppression. $\mathrm{N}$-cadherin is a major EMT marker, and as stated in previously publications, the EMT process can be reversed when $\mathrm{N}$-cadherin is deleted [ $\left.{ }^{12}\right]$. EMT activation is a common feature in advanced prostate cancer, is always involved in the neuroendocrine differentiation process, and is considered a marker of advanced cancer. EMT enforces immunosuppression and helps cancer cells escape immunosurveillance, which is known to play a major role in tumor invasion. Previous studies have suggested that EMT is activated in a number of malignant tumor cells, which results in the loss of typical characteristics of epithelial (Epi) cells (cell-cell junctions and apical-basal polarity) and the gain of mesenchymal (Mes) cell properties to promote migration and complete the invasion-metastasis cascade [ $\left.{ }^{28}\right]$. Therefore, whether immune checkpoint improvements simply occur as tumors become more malignant or whether they are regulated by EMT still requires further exploration. In our study, we demonstrated that $\mathrm{N}$-cadherin is an important hurdle for tumors overcome in order to generate a response to immunotherapy, as it regulates the expression of PD-L1/ID 0-1. Thus, N-cadherin can be considered a therapeutic target in immunosuppression. This is the core finding of our research.

Some studies have proposed that PD-1 blockade induces an incomplete rescue in cancers where its level is high $\left[{ }^{29}\right]$. Furthermore, other studies $\left[{ }^{30,31}\right]$ have demonstrated that IDO-1 and PD-L1 exert synergistic effects on tumor immune invasion, which is also in agreement with our data. This is the reason why combination treatment targeting both IDO-1 and PD-L1 has been explored; however, the best method would be to block the upstream pathway that regulates the expression of immunosuppression-related factors. Therefore, the first step of our research was validation of the role of $\mathrm{N}$-cadherin in the secretion of PD-L1/IDO-1.

In our study, we found that the highest levels of N-cadherin and PD-L1/IDO-1 expression are observed in small cell prostate cancer and that PD-L1/IDO-1 are expressed at higher levels in N-cadherin-positive tumors. Recently, a new molecular link between EMT-up-regulated PD-L1 expression and CD8+ tumorinfiltrating lymphocyte (TIL) immunosuppression was established in human lung cancer $\left[{ }^{[2}\right]$. EMT directly regulates the expression of PD-L1 and is associated with several other checkpoint ligands [32,33]. However, our research is the first to demonstrate that PD-L1 expression can be increased by the EMT marker $\mathrm{N}$-cadherin in prostate cancer. Kolijn et al. ${ }^{26}{ }^{26}$ also proposed that ID 0-1 can regulate $\mathrm{N}$-cadherin, which can prompt prostate cancer cells to secrete IDO-1, but they did not evaluate PD-L1 and provided only mRNA data, not protein data. We extended this finding, revealed the mutual regulation between EMT and immune checkpoint molecules, and verified this finding in patient-derived tissues, cells and a tumorbearing mouse model. We also found a coordinate expression between IDO-1 and PD-L1. This result led to a new hypothesis that combination treatment that targets both ID 0-1 and PD-L1 can abrogate the immunosuppressive effect elicited by IFN- $\triangle$ pathways that are active in the tumor microenvironment. However, the optimal therapy would still target upstream factors, and thus we provide evidence that $\mathrm{N}$ cadherin inhibition can disrupt PD-L1 and IDO-1 expression. We used a tumor-bearing mouse model to 
perform the treatment experiment. Our data shows that $\mathrm{N}$-cadherin deletion can terminate the immunosuppression induced by this PD-L1/IDO-1 in vivo model.

Furthermore, we also revealed how N-cadherin mediates the IFNGR/JAK1/STAT1 pathway, which decreases antitumor immunity by PD-L1/IDO-1 secretion. We also found that $\mathrm{N}$-cadherin can enhance JAK1 expression. Moreover, we observed that JAK1/STAT1 pathway activation was associated with higher expression of both PD-L1 and ID0-1. In contrast, JAK2 STAT3 was linked only to IDO-1 expression. The genomic loss of JAK1 occurred in some adenocarcinoma and CRPC cell lines [ $\left.{ }^{34}\right]$, which is the reason some cell lines, especially adenocarcinoma cell lines, with a deficient IFN囚 response failed to produce PD-L1/IDO-1. The expression of JAK1/2, STAT3, and PD-L1 increases during EMT, which has already been reported in lung cancer $\left[{ }^{19}\right]$, and in agreement with our research, JAK1 can be rescued in LNCap C1/C2 cell lines that express JAK1. Our data explain the active N-cadherin feedback loop between immunosuppression and EMT. These findings provide insights into the molecules and signaling pathways involved in the interaction between EMT and other immune processes, which will hopefully promote the development of different therapeutic strategies aimed at boosting or suppressing specific EMT functions, depending on the pathological context. Above all, we defined a positive feedback loop, which is initiated by N-cadherin, between EMT and immune checkpoint protein expression. Moreover, targeting $\mathrm{N}$-cadherin can significantly reverse immunosuppression, which is a very innovative discovery.

Although we used multiple model systems and human tissue, our study still had limitations. For example, the tumor xenograft model established in NSG mice lacks a tumor microenvironment composed of not only tumor cells but also lymphocytes. Additionally, IDO2 and TDO2 are also involved in Try degradation, but the roles of these two enzymes in EMT remain to be elucidated. The PC3 cell line is also controversial. PC3 is a special cell line, and usually, we do not classify it as SCC, even though it possesses some NE phenotypic characteristics, such as the expression of neuron-specific enolase (NSE). However, we sought to explore the association between $\mathrm{N}$-cadherin and immunosuppression, and thus we considered PC3 to be an $\mathrm{N}$-cad-positive cell line that expresses some NE markers. We also used two verified NE cell lines, LASCPC-01 and $\mathrm{NCl}-\mathrm{H} 660$, in this experiment to provide more evidence.

\section{Conclusion}

In summary, this study revealed a new immunotherapy biomarker in N-cadherin. N-cadherin knockout can significantly decrease the PD-L1 and IDO-1 expression induced by an IFN response. Moreover, N-cadherin can rescue the expression of JAK1, and the IFN response will up-regulate the immunosuppressive factors PD-L1/ID0-1; this finding reveals the molecular pathway of how N-cadherin initiates immune escape. In addition, our data also show how the an IFN response can enhance the EMT process through induction of PD-L1/ID0-1 expression.

\section{Abbreviations}

EMT: epithelial-to-mesenchymal transition 
SCC: small cell prostate cancer

Kyn: kynurenine

SCNC: small cell neuroendocrine prostate cancer

NED: neuroendocrine differentiation

PCa: prostate cancer

PD-L1: Programmed death ligand-1

IDO-1: indole amine 2,3-dioxygenase

NSE: neuron-specific enolase

H\&E: hematoxylin and eosin

\section{Declarations}

Ethical Approval and Consent to participate: The mice experiment was approved by Duke ethical association

Consent for publication: Not applicable

Availability of supporting data: The raw data can be acquired from corresponding author Qiang Wei (arfuzinss@sina.com)

Competing interests: There are no competing interests within authors.

Funding: This study was supported by the National Natural Science Foundation of China (Grant No. $81370855,81300627,81702536,81770756$ and 81200551) for L.Y. and Q.W., This study was supported by the National key research and development program of China (Grant No. SQ2017YFSF090096) Q.W., the Prostate Cancer Foundation Young Investigator Award 2013, Foundation of Science \&Technology Department of Sichuan Province (Grant No. 2015SZ0230, 2013SZ0006 and 2013SZ0093) for Q.W., Programs from Science and Technology Department of Sichuan Province (Grant No. 2018JY0089 and 2017HH0063) for Q.W., Young Investigator Award of Sichuan University 2017, and the Scientific Research Project of Health Department of Sichuan Province (No. 120203) for L.Y..

Authors' contributions: Y.S., L.Y., H. X. and Q.W. contributed to the conception of the study; Y.S., L.Y., R.R., J. H. and J.G. performed the experiment; Y.S., L.Y., R.R., J. H. and J.G. contributed significantly to analysis and manuscript preparation; Y.S., J.H., R.R. and J.G. performed the data analyses and wrote the manuscript; Y.S., R.R., H.X., J.G. and Q.W. helped perform the analysis with constructive discussions. 
Acknowledgements: Yi Sun and Ju Guo contributed equally to this work and should share the co-first author.

\section{References}

1. Nelson EC, Cambio AJ, Yang JC, Ok JH, Lara PN Jr, Evans CP. Clinical implications of neuroendocrine differentiation in prostate cancer. Prostate Cancer Prostatic Dis 2007; 10 (1), 6-14.

2. Zong Y, Goldstein AS. Adaptation or selection-mechanisms of castration- resistant prostate cancer. Nat Rev Urol 2013; 10 (2), 90-98.

3. Lipianskaya J, Cohen A, Chen CJ, Hsia E, Squires J, Li Z, Zhang Y, Li W, Chen X, Xu H, Huang J. Androgen-deprivation therapy-induced aggressive prostate cancer with neuroendocrine differentiation. Asian J Androl 2014; 16 (4), 541-544.

4. Julie R Brahmer, Scott S Tykodi, Laura Q M Chow, Wen-Jen Hwu, Suzanne L Topalian, Patrick Hwu, Charles G Drake, Luis H Camacho, John Kauh, Kunle Odunsi, Henry C Pitot, Omid Hamid, Shailender Bhatia, Renato Martins, Keith Eaton, Shuming Chen, Theresa M Salay, Suresh Alaparthy, Joseph F Grosso, Alan J Korman, Susan M Parker, Shruti Agrawal, Stacie M Goldberg, Drew M Pardoll, Ashok Gupta, Jon M Wigginton. Safety and Activity of anti-PD-L1 Antibody in Patients With Advanced Cancer. N Engl J Med. 2012 Jun 28;366(26):2455-65

5. Hamid O, Caroline Robert, Adil Daud, F Stephen Hodi, Wen-Jen Hwu, Richard Kefford, Jedd D Wolchok, Peter Hersey, Richard W Joseph, Jeffrey S Weber, Roxana Dronca, Tara C Gangadhar, Amita Patnaik, Hassane Zarour, Anthony M Joshua, Kevin Gergich, Jeroen Elassaiss-Schaap, Alain Algazi, Christine Mateus, Peter Boasberg, Paul C Tumeh, Bartosz Chmielowski, Scot W Ebbinghaus, Xiaoyun Nicole Li, S Peter Kang, Antoni Ribas. Safety and Tumor Responses With Lambrolizumab (anti-PD-1) in Melanoma. N Engl J Med. 2013 Jul 11;369(2):134-44

6. Amarnath S, Mangus CW, Wang JC, Wei F, He A, Kapoor V, Foley JE, Massey PR, Felizardo TC, Riley JL, Levine BL, June CH, Medin JA, Fowler DH. The PDL1-PD1 axis converts human TH1 cells into regulatory T cells. Sci Transl Med 2011; 3(111), 111ra120-111ra120.

7. Takikawa O, Kido R, Hayaishi O. Tryptophan degradation in mice initiated by indoleamine 2,3dioxygenase. J Biol Chem 1986; 261, 3648-3653.

8. Munn DH, Sharma MD, Baban B, Harding HP, Zhang Y, Ron D, Mellor AL. GCN2 kinase in T cells mediates proliferative arrest and anergy induction in response to indoleamine 2,3-dioxygenase. Immunity 2005; 22 (5), 633-642.

9. Ock CY, Kim S, Keam B, Kim M, Kim TM, Kim JH, Jeon YK, Lee JS, Kwon SK, Hah JH, Kwon TK, Kim DW, Wu HG, Sung MW, Heo DS. PD-L1 expression is associated with epithelial-mesenchymal transition in head and neck squamous cell carcinoma. Oncotarget 2016; 7 (13), 15901-15914.

10. Asgarova A, Asgarov K, Godet Y, Peixoto P, Nadaradjane A, Boyer-Guittaut M, Galaine J, Guenat D, Mougey V, Perrard J, Pallandre JR, Bouard A, Balland J, Tirole C, Adotevi O, Hendrick E, Herfs M, Cartron PF, Borg C, Hervouet E. PD-L1 expression is regulated by both DNA methylation and NF-kB during EMT signaling in non-small cell lung carcinoma. Oncolmmunology 2018; 7(6), e1423170. 
11. Wang Y, Wang H, Zhao Q, Xia Y, Hu X, Guo J. PD-L1 induces epithelial-to-mesenchymal transition via activating SREBP-1c in renal cell carcinoma. Med Oncol 2015; 32(8), 212.

12. Hiroshi Tanaka, E.K., Chau P Tran, Hideyo Miyazaki, Joyce Yamashiro, Tatsuya Shimomura, Ladan Fazli, Robert Wada, Jiaoti Huang, Robert L Vessella, Jaibin An, Steven Horvath, Martin Gleave, Matthew B Rettig, Zev A Wainberg \& Robert E Reiter. Monoclonal antibody targeting of N-cadherin inhibits prostate cancer growth, metastasis and castration resistance. Nature Medicine 2010. 16, 1414-1421.

13. Qiang Danga, L.L., Hongjun Xie, Dalin He, Jiaqi Chen, Wenbing Song, Luke S. Chang, Hong-Chiang Chang, Shuyuan Yehb, Chawnshang Chang. Anti-androgen enzalutamide enhances prostate cancer neuroendocrine (NE) differentiation via altering the infiltrated mast cells / androgen receptor (AR) / miRNA32 signals. Mol Onco 2015. 9, 1241-1251.

14. Kolijn K, Verhoef El, Smid M, Böttcher R, Jenster GW, Debets R, van Leenders GJLH. EpithelialMesenchymal Transition in Human Prostate Cancer Demonstrates Enhanced Immune Evasion Marked by ID01 Expression. Cancer Res 2018; 78(16), 4671-4679.

15. Janielle P. Maynard, O.E., Ibrahim Kulac, Javier A. Baena-Del Valle, Angelo M. De Marzo, and Karen S. Sfanos. IL8 Expression Is Associated with Prostate Cancer Aggressiveness and Androgen Receptor Loss in Primary and Metastatic Prostate Cancer. Mol Cancer Res 2020. 18(1), 153-169.

16. Wu ZH, Lin C, Liu CC, Jiang WW, Huang MZ, Liu X, Guo WJ. MiR-616-3p promotes angiogenesis and EMT in gastric cancer via the PTEN/AKT/mTOR pathway. Biochem Biophys Res Commun 2018; 501(4), 1068-1073.

17. Park JW, Lee JK, Witte ON. FOXA is a sensitive and specific marker for small cell neuroendocrine carcinoma of the prostate. Mod Pathol 2017; 30, 1262-1272.

18. John K. Lee, J.W.P., Bryan A. Smith, Jung Wook Park, Tanya Stoyanova, Erin F. McCaffrey, Robert Baertsch, Artem Sokolov, Justin G. Meyerowitz, Colleen Mathis, Donghui Cheng, Joshua M. Stuart, Kevan M. Shokat, W. Clay Gustafson, Jiaoti Huang, and Owen N. Witte. N-Myc Drives Neuroendocrine Prostate Cancer Initiated from Human Prostate Epithelial Cells. Cancer Cell 2016. 29 (4), 536-547.

19. Shen M, Xu Z, Xu W, Jiang K, Zhang F, Ding Q, Xu Z, Chen Y. Inhibition of ATM reverses EMT and decreases metastatic potential of cisplatin resistant lung cancer cells through JAK/STAT3/PDL1 pathway. J Exp Clin Cancer Res 2019; 38 (1), 149-162.

20. Yi Sun, Jianzhong Ai, Xi Jin, Liang-Ren Liu, Tian-Hai Lin, Hang Xu, Qiang Wei and Lu Yang. IL-8 protects prostate cancer cells from GSK-3ß-induced oxidative stress by activating the mTOR signaling pathway. The Prostate 2019. 79 (10), 1180-1190.

21. McKeithen D, Graham T, Chung LW, Odero-Marah V. Snail transcription factor regulates neuroendocrine differentiation in LNCaP prostate cancer cells. Prostate Cancer Prostatic Dis 2010; 70 (9), 982-992.

22. Conteduca V, Aieta M, Amadori D, De Giorgi U. Neuroendocrine differentiation in prostate cancer: current and emerging therapy strategies. Crit Rev Oncol Hematol 2014; 92(1), 11-24. 
23. Danza G, Di Serio C, Rosati F, Lonetto G, Sturli N, Kacer D, Pennella A, Ventimiglia G, Barucci R, Piscazzi A, Prudovsky I, Landriscina M, Marchionni N, Tarantini F. Notch signaling modulates hypoxia-induced neuroendocrine differentiation of human prostate cancer cells. Mol Cancer Res 2012; 10 (2), 230-238.

24. Luo Y, Lan L, Jiang YG, Zhao JH, Li MC, Wei NB, Lin YH. Epithelial-mesenchymal transition and migration of prostate cancer stem cells is driven by cancer-associated fibroblasts in an HIF1alpha/beta-catenin-dependent pathway. Mol Cells 2013; 36 (2), 138-144.

25. Marhold M, T.E., El-Gazzar A, Heller G, Spittler A, Horvat R, Krainer M1, Horak P. HIF-1alpha regulates mTOR signaling and viability of prostate cancer stem cells. Mol Cancer Res 2014. 13 (3), 556-64.

26. Y. Zhang R.A. Weinberg. Epithelial-to-mesenchymal transition in cancer: complexity and opportunities. Front Med 2018, 12: 361-373.

27. Banzola I, Mengus C, Wyler S, Hudolin T, Manzella G, Chiarugi A, Boldorini R, Sais G, Schmidli TS, Chiffı G, Bachmann A, Sulser T, Spagnoli GC, Provenzano M. Expression of indoleamine 2,3Dioxygenase induced by IFN-y and TNF-a as Potential Biomarker of Prostate cancer Progression. Front Immunol 2018. 29, 1051.

28. Rama Soundararajan, J.J.F., Jessica M. Konen, Stacy Moulder , Xiang Zhang, Don L. Gibbons , Navin Varadarajan, Ignacio I. Wistuba, Debasish Tripathy, Chantale Bernatchez, Lauren A. Byers, Jeffrey T. Chang, Alejandro Contreras, Bora Lim , Edwin Roger Parra, Emily B. Roarty, Jing Wang, Fei Yang, Michelle Barton, Jeffrey M. Rosen , and Sendurai A. Mani. Targeting the Interplay between Epithelialto-Mesenchymal-Transition and the Immune System for Effective Immunotherapy. Cancers 2019. 11, 714.

29. M. M. Gubin, X. Zhang, H. Schuster, E. Caron, J. P. Ward, T. Noguchi, Y. Ivanova, J. Hundal, C. D. Arthur, W.-J. Krebber, G. E. Mulder, M. Toebes, M. D. Vesely, S. S. K. Lam, A. J. Korman, J. P. Allison, G. J. Freeman, A. H. Sharpe, E. L. Pearce, T. N. Schumacher, R. Aebersold, H.-G. Rammensee, C. J. M. Melief, E. R. Mardis, W. E. Gillanders, M. N. Artyomov, R. D. Schreiber, Checkpoint blockade cancer immunotherapy targets tumour-specific mutant antigens. Nature 2014. 515, 577-581

30. Derek A. Wainwright Alan L. Chang, Mahua Dey, Irina V. Balyasnikova, Chung Kwon Kim, Alex Tobias, Yu Cheng, Julius W. Kim, Jian Qiao, Lingjiao Zhang, Yu Han, and Maciej S. Lesniak. Durable therapeutic efficacy utilizing combinatorial blockade against IDO, CTLA-4 and PD-L1 in mice with brain tumors. Clin Cancer Res 2014, 20 (20): 5290-5301.

31. Douglas B. Johnson Jennifer Bordeaux, Ju Young Kim, Christine Vaupel, David L. Rimm, Thai H. Ho, Richard W Joseph, Adil I. Daud, Robert M. Conry, Elizabeth M. Gaughan, Leonel F. Hernandez-Aya, Anastasios Dimou, Pauline Funchain, James Smithy, John S. Witte, Svetlana B. Mckee, Jennifer Ko, John M. Wrangle, Bashar Dabbas, Shabnam Tangri, Jelveh Lameh, Jeffrey Hall, Joseph Markowitz, Justin M. Balko, and Naveen Dakappagari. Quantitative Spatial Profiling of PD-1/PD-L1 Interaction and HLA- DR/ID0-1 Predicts Improved Outcomes of anti-PD-1 Therapies in Metastatic Melanoma. Clin Cancer Res 2018, 24 (21): 5250-5260. 
32. Chen L, Gibbons DL, Goswami S, Cortez MA, Ahn YH, Byers LA, Zhang X, Yi X, Dwyer D, Lin W, Diao L, Wang J, Roybal J, Patel M, Ungewiss C, Peng D, Antonia S, Mediavilla-Varela M, Robertson G, Suraokar M, Welsh JW, Erez B, Wistuba II, Chen L, Peng D, Wang S, Ullrich SE, Heymach JV, Kurie JM, Qin FX. Metastasis is regulated via microRNA-200/ZEB1 axis control of tumour cell PD- L1 expression and intratumoral immunosuppression. Nat Commun 2014. 5, 5241.

33. Loi S, Dushyanthen S, Beavis PA, Salgado R, Denkert C, Savas P, Combs S, Rimm DL, Giltnane JM, Estrada MV, Sánchez V, Sanders ME, Cook RS, Pilkinton MA, Mallal SA, Wang K, Miller VA, Stephens PJ, Yelensky R, Doimi FD, Gómez H, Ryzhov SV, Darcy PK, Arteaga CL, Balko JM. RAS/MAPK Activation Is Associated with Reduced Tumor-Infiltrating Lymphocytes in Triple-Negative Breast Cancer: Therapeutic Cooperation Between MEK and PD-1/PD-L1 Immune Checkpoint Inhibitors. Clin Cancer Res 2016. 22, 1499-1509.

34. Dunn GP, Sheehan KC, Old LJ, Schreiber RD. IFN Unresponsiveness in LNCaP Cells Due to the Lack of JAK1 Gene Expression. Cancer Res 2005. 65, 3447-3453.

\section{Figures}

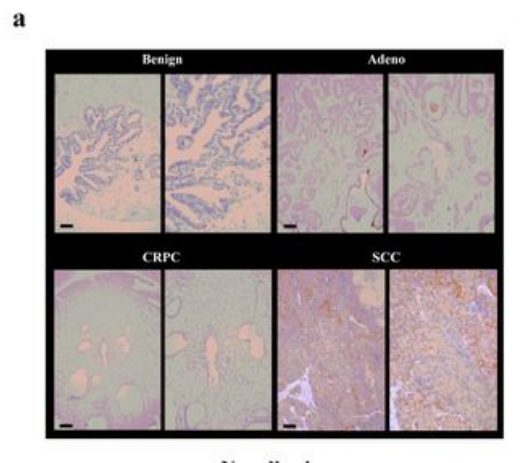

$\mathrm{N}$-cadherin

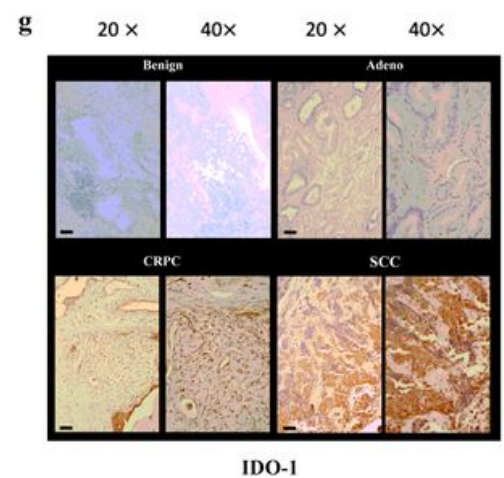

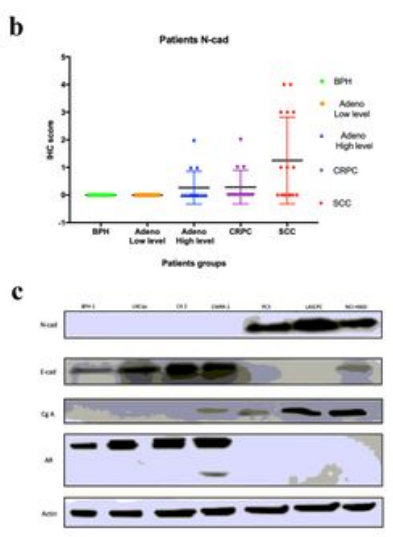
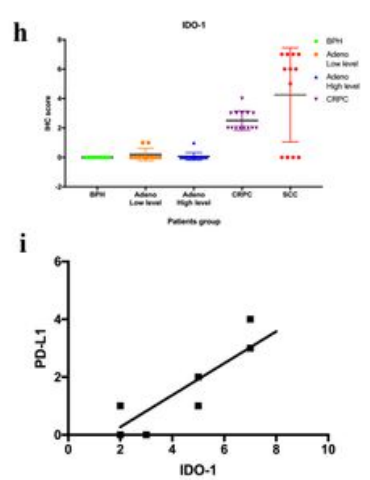

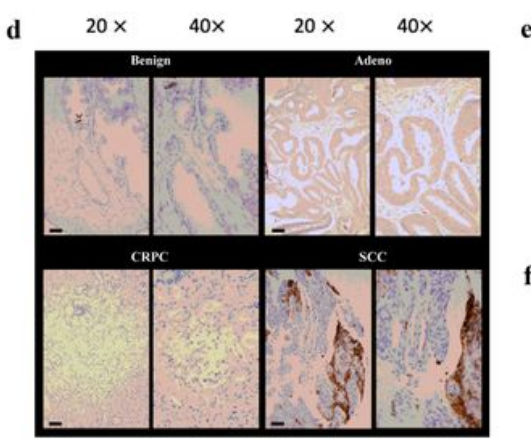

PD-L1
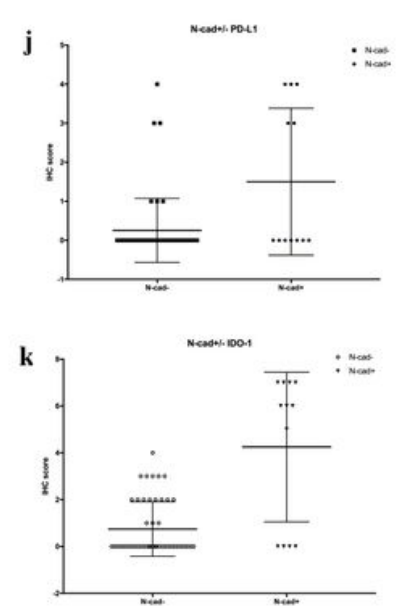

Figure 1

Expression of $\mathrm{N}$-cadherin (N-cad), indoleamine-2,3-dioxygenase (IDO-1) and programmed death receptor-1 (PD-L1) in patient tissue samples and in cell lines. (a) Immunohistochemical staining for $\mathrm{N}$-cad expression in benign, adenocarcinoma, castration-resistant prostate cancer (CRPC) and small cell 
prostate cancer (SCC) tissue samples. (b) Immunohistochemistry (IHC) scores for N-cad expression in different tumors. (c) Expression of N-cad and E-cadherin (E-cad) in different cell lines. (d) Immunohistochemical staining for PD-L1 expression in different tumors. (e) IHC scores for PD-L1 expression in different tumors. (f) Expression of PD-L1 in different cell lines. (g) Immunohistochemical staining for PD-L1 expression in different tumors. (h) IHC scores of different tumors. (i) Linear relationship analysis of IDO-1 expression and PD-L1 expression. ( $j, k$ ) Expression of PD-L1 and IDO-1 in Ncad-positive and N-cad-negative samples. (I) Expression of Chromogranin-A ( $\mathrm{Cg} \mathrm{A}$ ) and neuron-specific enolase (NSE) in different cell lines.

a

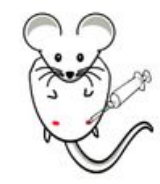

e
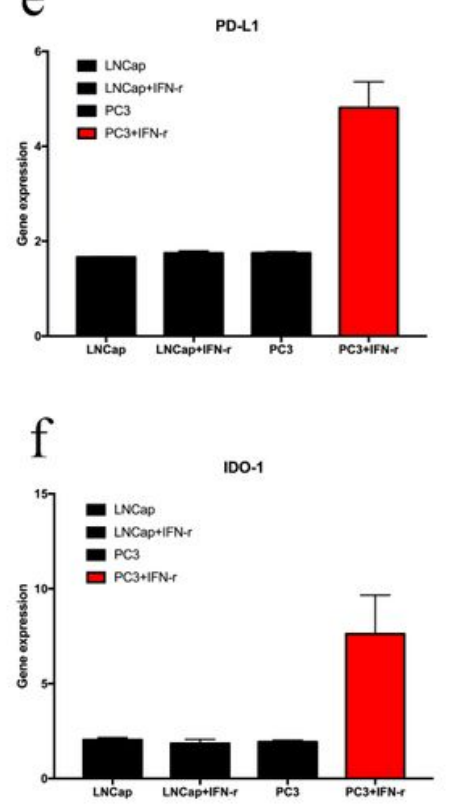

$\mathrm{C}$

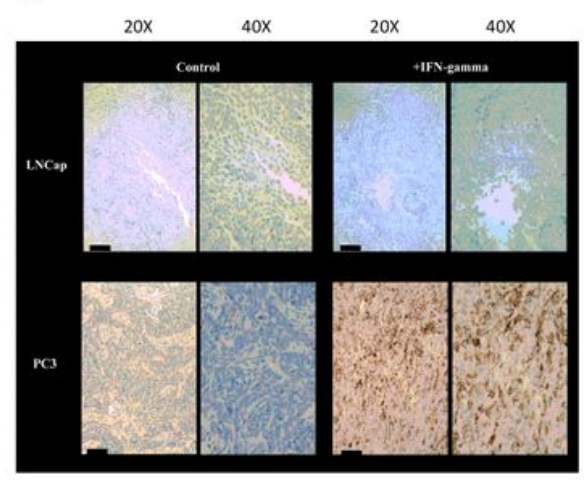

PD-L1

$\mathrm{g}$

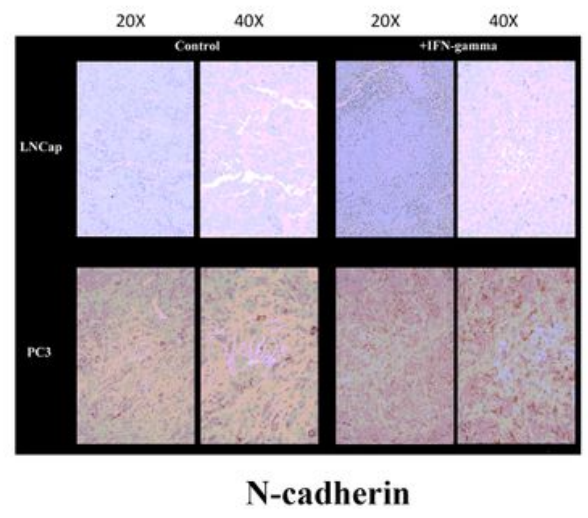

$\mathrm{d}$

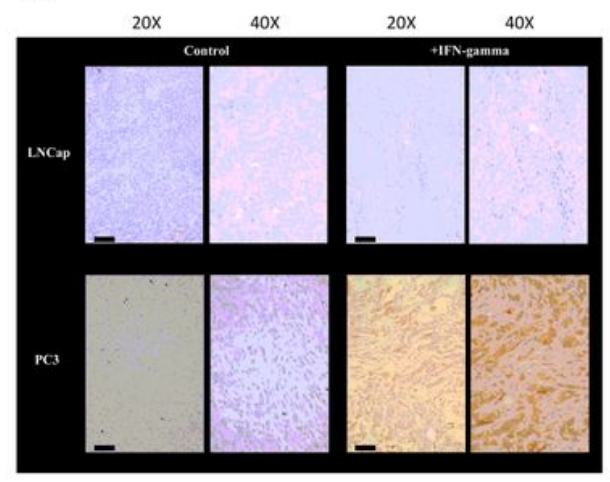

IDO-1

$\mathrm{h}$

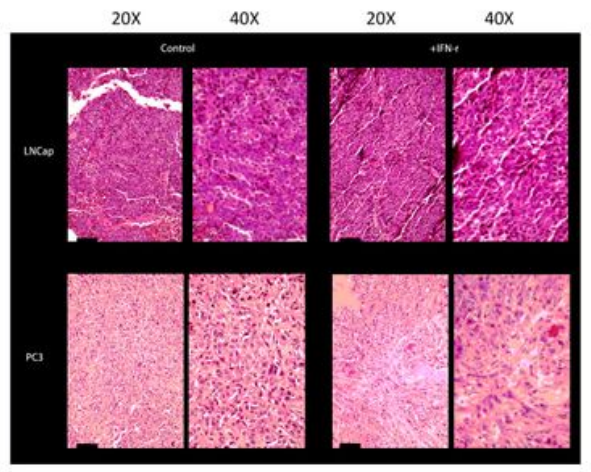

H\&E

Figure 2

Expression of N-cad, IDO-1 and PD-L1 in mouse tumors. (a) Tumor implantation site. (b) Flow cytometric analysis of mouse tumor cells after tissue dissociation. (c) Immunohistochemical staining for PD-L1 expression in different tumors. (d) Immunohistochemical staining for ID0-1 expression in different tumors. (e) mRNA expression of PD-L1 in different tumors after tissue dissociation and RNA extraction. (f) mRNA expression of IDO-1 in different tumors. (g) Immunohistochemical staining for ID 0-1 expression in different tumors. (h) Hematoxylin and eosin (H\&E) staining of mouse tissues. 


\section{a}

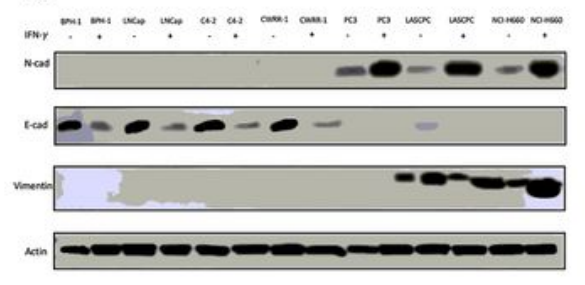

$\mathrm{b}$

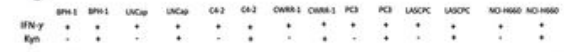

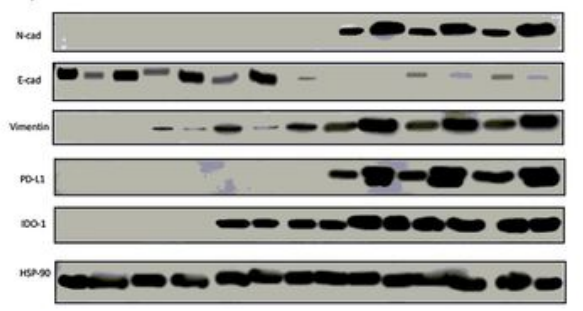

c

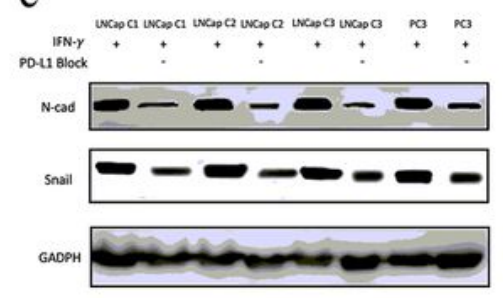

d
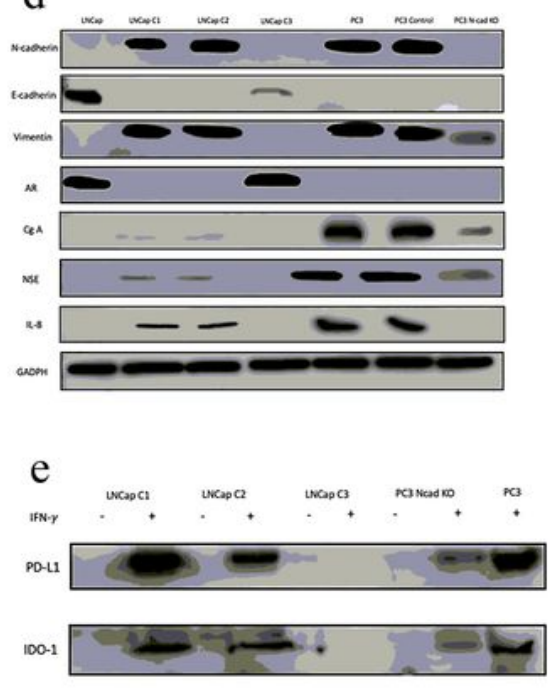

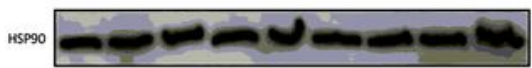

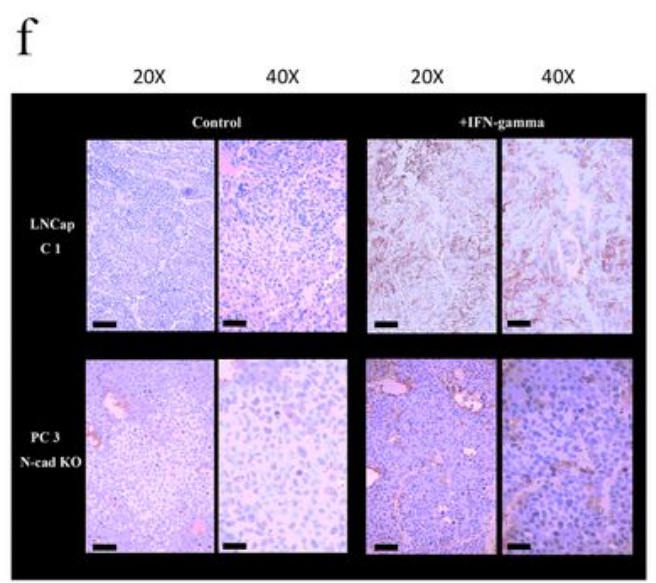

PD-L1

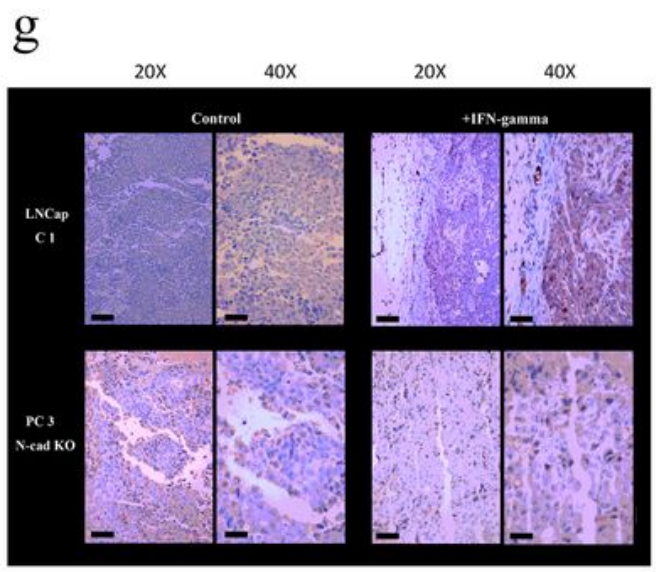

IDO-1

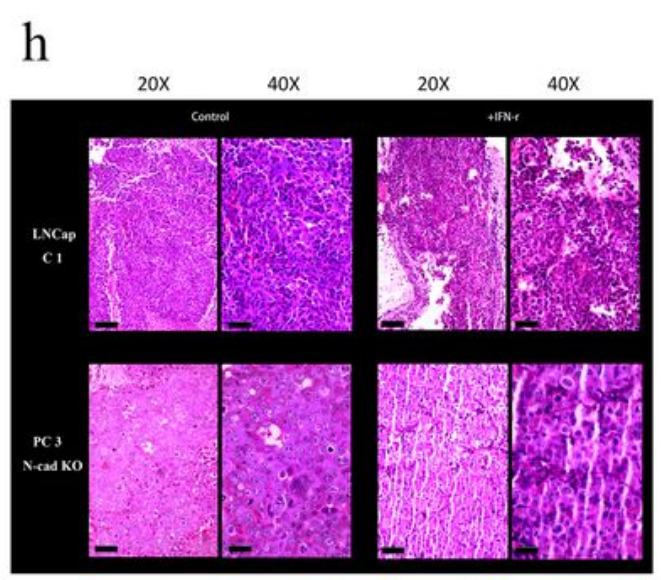

H\&E

\section{Figure 3}

Evaluation of the impacts of N-cad on the expression of PD-L1 and IDO-1. (a) Changes in the levels of Ncad in cell lines after treatment with interferon gamma (IFN-C). (b) Changes in PD-L1, IDO-1 and EMT markers expression after treatment with kynurenine (Kyn). (c) Changes in EMT markers levels after treatment with PD-L1 neutralizing Antibody. (d) EMT and neuroendocrine (NE) phenotypes in N-cadoverexpressing cells and N-cad knockout (N-cad KO) cells. (e) PD-L1 and ID 0-1 expression in N-cad- 
overexpressing cells and N-cad KO cells after treatment with IFN-囚. (f) Immunohistochemical staining for PD-L1 expression in different tumors. (g) Immunohistochemical staining for ID0-1 expression in different tumors. (h) Hematoxylin and eosin (H\&E) staining of mouse tissues.

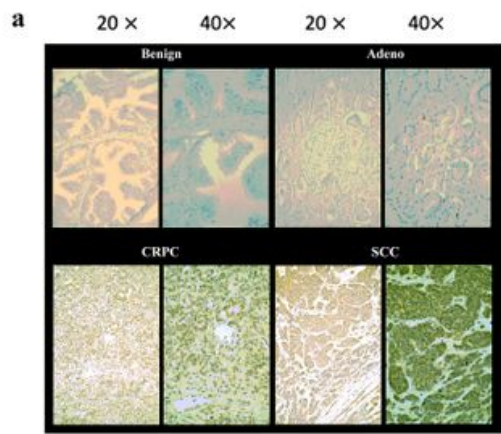

IFNGR1
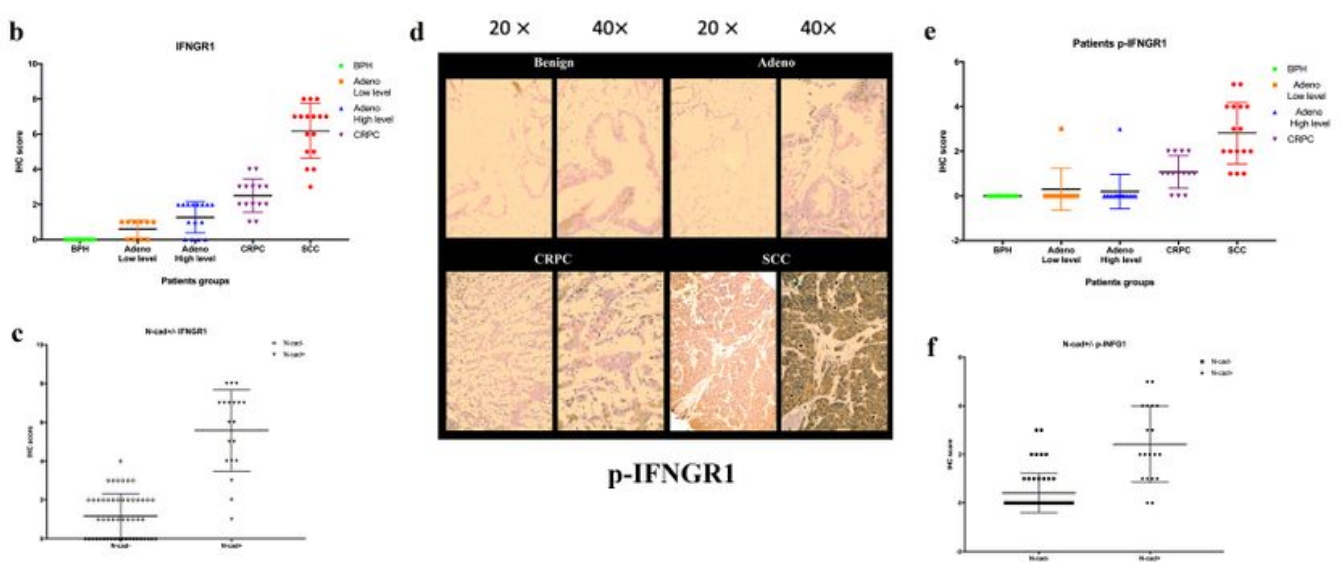

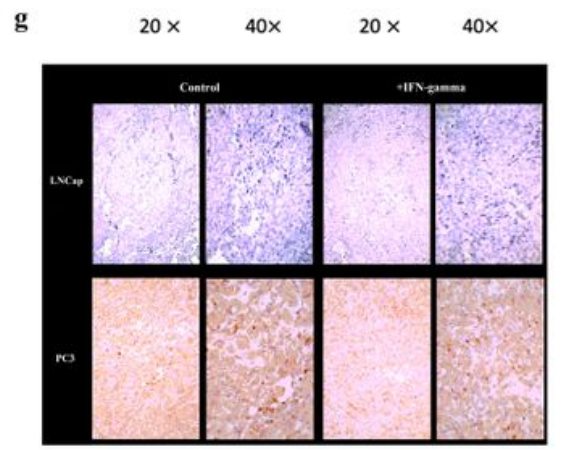

IFNGR1 h

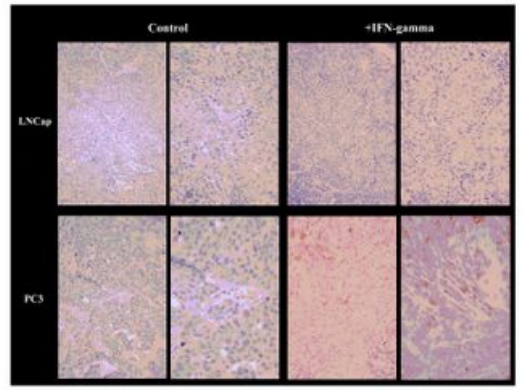

p-IFNGR1

Figure 4

Exploration of PD-L1 and IDO-1 expression regulated by interferon gamma receptor 1 (IFNGR1). (a) Immunohistochemical staining for IFNGR1 expression in benign, adenocarcinoma, CRPC and SCC tissue samples. (b) IHC scores for IFNGR1 expression in different tumors. (c) IFNGR1 expression in N-cadpositive and N-cad-negative cases. (d) Immunohistochemical staining for $\mathrm{p}$-IFNGR1 expression in benign, adenocarcinoma, CRPC and SCC tissue samples. (e) IHC scores for p-IFNGR1 expression in different tumors. (f) p-IFNGR1 expression in N-cad-positive and N-cad-negative cases. (g) Immunohistochemical staining for IFNGR1 expression in mouse tissue samples. (h) Immunohistochemical staining for $\mathrm{p}$ IFNGR1 expression in mouse tissue samples. (i) Expression of IFNGR1/p-IFNGR1 in different cell lines. 

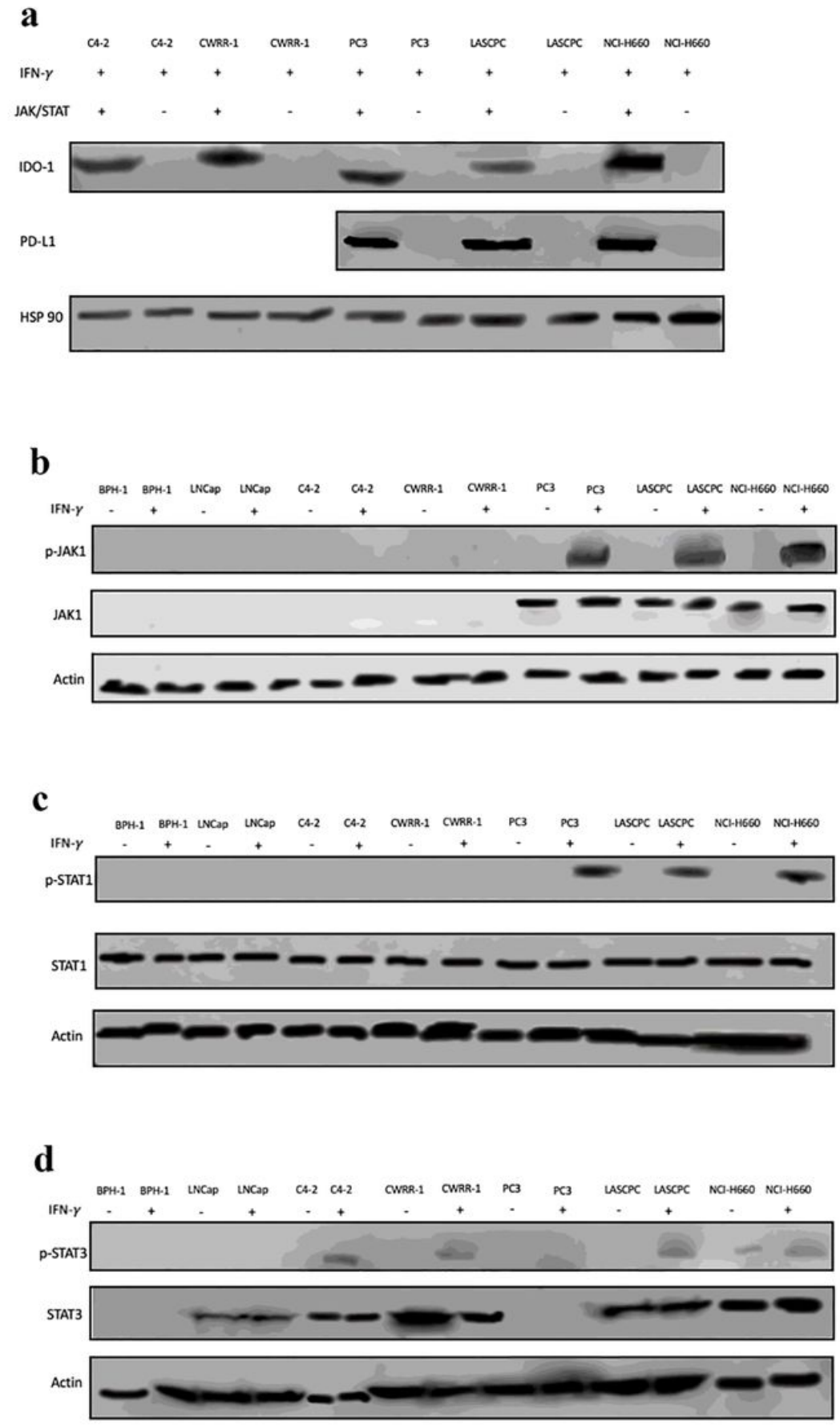
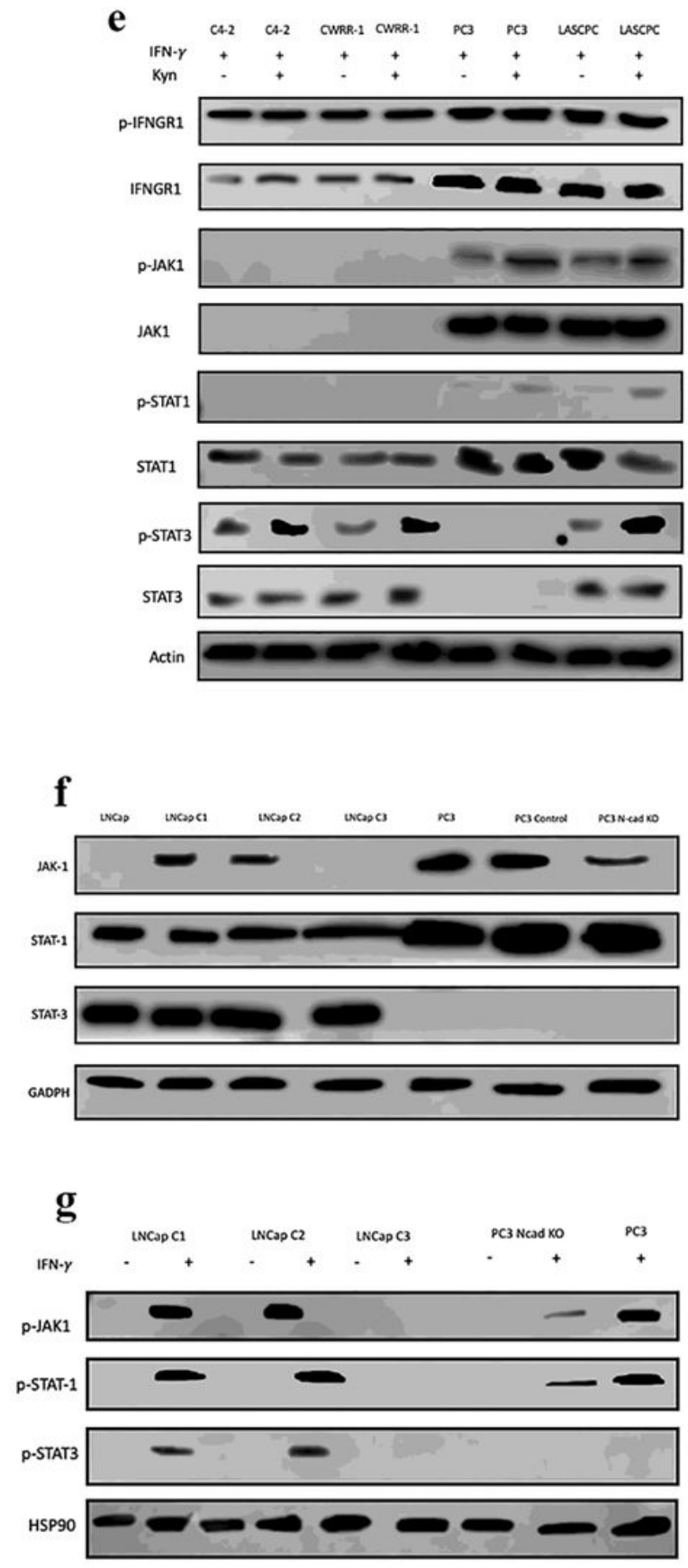

\section{Figure 5}

N-cadherin regulates PD-L1 and IDO-1 expression by impacting the JAK/STAT pathway. (a) Expression of PD-L1 and IDO-1 after JAK/STAT pathway blockade. (b, c, d) JAK/STAT pathway activation and PDL1/ID0-1 expression. (e) Activation of the IFNGR/JAK/STAT pathway after treatment with Kyn. (f) The expression of JAK1/JAK3 in N-cad-overexpressing cells and N-cad KO cells. (g) The activation of the IFNGR/JAK/STAT pathway in N-cad-overexpressing cells and N-cad KO cells after treatment with IFN- $\mathrm{Z}$.

\section{Supplementary Files}


This is a list of supplementary files associated with this preprint. Click to download.

- SupplementFigure4.tif

- SupplementFigure4.tif

- SupplementFigure4.tif

- SupplementFigure3.tif

- SupplementFigure3.tif

- SupplementFigure3.tif

- SupplementFigure2.tif

- SupplementFigure2.tif

- SupplementFigure2.tif

- SupplementFigure1.tif

- SupplementFigure1.tif

- SupplementFigure1.tif 